\title{
A NEW TREND IN PHOTOPOLYMERS BASED ON THE COMMANDER/SOLDIER CONCEPT
}

\author{
KUNIHIRO ICHIMURA \\ Research Laboratory of Resources Utilization, \\ Tokyo Institute of Technology, \\ 4259 Nagatsuta, Midori-ku, Yokohama 226, Japan
}

\begin{abstract}
Some photosensitive molecular systems are triggered by the photon absorption leading to the structural change of commander molecules which initiate the consequent processes consisting of structural as well as orientational modification of a large number of soldier molecules. The nove1 commander/soldier systems include photoinduced phase changes of liquid crystals, surface-mediated liquid crystal alignment photoregulation and photoregulation of the helical sense of a poly(isocyanate). Further discussions are made on the molecular amplification of photopolymer systems.
\end{abstract}

\section{Introduction}

Photochemical processes in polymeric systems give birth to versatile photopolymers displaying various functionalities as a result of marked alteration of their chemical as well as physical properties. In this context, it is appropriate to classify poly(vinyl cinnamate), one of the representative photopolymers, and rhodopsin, a photosensitive biopolymer, in the same category since both polymers modify their states owing to the photon absorption. But why does it sound rather unfamiliar to say so? Because of a quite different photofunctionalities? Or because of the highly ordered macromolecular skeleton of the biopolymer? The following point should be stressed to clarify the intrinsic difference between these exemplified photopolymers. In the case of poly(vinyl cinnamate), the photochemistry leading to the photocrosslinkage takes place locally at cinnamate residues in the polymer solid matrix so that no essential modification of the three-dimensional structure of the macromolecules is induced. On the contrary, the geometrical photoisomerization of the cis-11-retinylidene residue brings about the confor-

Received April 6, 1995 
mational alteration of opsin which is just the beginning of the subsequent conjugated processes in the visual sensory systems.

The major concern of this paper is to present a novel concept concerning the causality of photochemical processes in photopolymer systems and to discuss the adaptability of this concept to various polymeric systems triggered by photon absorption, focusing on the nature of molecular amplification [1].

\section{Commander-soldier concept}

At the beginning of our systematic studies on the photoinduced regulation of liquid crystal (LC) alignment by substrate surfaces covered with photochromic molecular layers [2], we proposed to call such surfaces "command surfaces", stressing the fact that on the average one photochromic molecule attached to a surface is able to rearrange the orientation of about $10^{4}$ LC molecules. Here, the state of molecular aggregation is regulated by specific surface molecules to cause a phase change. It becomes rather inappropriate in this system to call photoactive molecules like azobenzenes photochromic since their functionality is not based on the color change any longer, but on how to start the molecular reorientation of a lot of LC molecules. It is noteworthy to remember that photochromism is defined as a photochemical phenomenon demonstrating reversible absorption spectral change based on a molecular structural alteration. In this respect, photoactive molecules triggering LC alignment is reasonably termed as "commander molecules" while LC is referred to as "soldier molecules" on account of the manner of their action.

Photon absorption plays a crucial role in the action of commander molecules because the input of molecular information carried by photons can be controlled readily in time and space and brings about subsequent molecular events of soldier molecules to cause the modification of material properties. This means that great varieties of photopolymers can be derived from a kind of commander-soldier systems made of polymeric materials. The followings are considered to be typical examples of photopolymers based on the commandersoldier concept.

\section{Commander-soldier polymeric systens}

Photosensitive liquid crystal polymers

Mesophase changes have been known to be induced photochemically by dissolving photochromic molecules in low-mass LC phases [3]. They involve the photoinduced phase changes between nematic/isotropic, cholesteric/isotropic 


\section{J. Photopolym. Sci. Technol., Vol.8, No.2, 1995}

and smectic/nematic phases. Cholesteric pitch bands are regulated photochemically by dissolved azobenzene [4] while the polarization flop of ferroelectric LCs is induced by the photoisomerization of doped azobenzenes [5]. In these LC systems, commander molecules are photochromic compounds like azobenzenes dissolved in LCs which behave as soldiers to demonstrate the phase changes. The UV-light-induced phase change of nematic LC has been extended by dissolving azo-commander molecules in or attaching them to polymeric backbones [6].

An alternate system consisting of LC polymers bearing azo-groups in their side chains is based on polarization photochromism $[7,8]$. Exposure of a layer of LC azo-polymers in their glassy state to linearly-polarized blue light for the $n, \pi^{*}$-transition of azo-chromophores causes the reorientation of the chromophores to result in a modification of anisotropic properties like dichroism and birefringence. The birefringence is markedly enhanced by the reorientation of photoinactive mesogenic units which is generated by the azounits as a commander $[9,10]$. The reorientation process results from an angular-dependent photoselection within the steady state of the photoisomerization of the azo-groups. On the other hand, the extent of the reorientation of non-photochromic groups in an LC polymer matrix is temperature-dependent [11] and influenced by the nature of LC polymers [8].

The remarkable photo-optical effect of these LC polymers is derived from the orientational modification of non-photochromic mesogens in their side chain. This supports the adaptability of the commander-soldier concept.

\section{Command surfaces derived from photochromic polymers}

The alignment of nematic LC can be regulated photochemically by photochromic molecules attached to a topmost surface of substrate plate. There are two types of photoregulative modes. The regulation of out-of-plane alignment is achieved by the $\mathrm{E} / \mathrm{Z}$ photoisomerization of azobenzenes on a silica plate surface $[2,12]$ while in-plane alignment is regulated by the irradiation of surface azobenzenes with linearly polarized light [13]. The in-plane alignment photoregulation can be performed by using thin films of polymers doped [13] or substituted with azobenzenes [14].

The reversible out-of-plane alignment alteration between homeotropic (perpendicular) and planar (parallel) modes is carried out by the $\mathrm{E} / \mathrm{Z}$ photoisomerization of azo-chromophores of a polymer thin film [14]. The molecular structure of azobenzenes attached to polymers should be designed so that a thin film of an azo-polymer causes a homeotropic alignment before UV 
irradiation. Substitution with a p-alkyl residue with a hydrophobic character is an effective way to bring about a homeotropic alignment when the azochromophore is E-isomer [15].

Reversible in-plane alignment has been attained by employing polymer thin films doped with dichroic dyes $[13,16]$ and thin films of poly(methacrylates) bearing azobenzene groups in the side chains [15]. The azimuthal alignment regulation is performed also by linearly polarized irradiation of a monolayer of a polyimide having p-cyanoazobenzene groups [17]. This fact indicates unequivocally that the alignment of LC molecules is determined by azo-units localized at a topmost surface of a substrate plate. The LC photoalignment arises from the uniaxial reorientation of azo-chromophores on a surface.

A homogeneous alignment is gained by polarized irradiation of a film of the photocrosslinkable polymer, poly(vinyl cinnamate) and its derivative $[18,19]$. This phenomenon is noteworthy from a viewpoint of the commandersoldier concept since such a conventional photoresist displays a unique photofunctionality by a combination with LC soldier molecules. Although the aligning mechanism has been thought to be due the angular-selective photodimerization of the cinnamate groups [18], further studies are needed. A silica surface covered with monolayered cinnamate residues is active for the in-plane alignment photoregulation [20], suggesting the involvement of the geometrical photoisomerization. Lateral attachment of cinnamate groups to polymer backbones is also an effective way to the in-plane alignment regulation [22].

\section{Helical structure as a soldier}

Poly(isocyanates) have been known to form a helical conformation. Being different from polypeptide chains, the helical sense of this type polymer, which has no asymmetric carbon atom in the main chain, is governed by copolymerization of an optical active isocyanate monomer. Interestingly, even a low content of an asymmetric monomer in an isocyanate copolymer determines the helical sense in a solution state. Consequently, according to Green and his coworkers the optical active isocyanate monomer has been referred to as sergeant monomer while an optically inactive monomer has been called soldier monomer [21]. Recent work has revealed that the helical sense of this type of polymers is photoregulated by introducing optically active azobenzene units in the side chain [22]. The optical rotatory dispersion as well as the circular dichroism of a solution of the polymer is modified as a result of the 
photoisomerization of the azo-chromophore. Thus, the azobenzene unit and the other part of the polymer chain are called a commander and soldier, respectively.

\section{Molecular amplification in command molecular systems}

As the examples shown in the preceding section tell us, a prominent feature of photopolymeric systems based on the commander-soldier concept involves the molecular amplification of photonic information. Various modes of molecular amplification can be proposed, as summarized in Fig. 1. For instance, the photoinduced phase change of LC polymers belongs to the type-1 of the molecular amplification since it consists of two molecular processes involving the photoconversion of azobenzene as a commander (C) into the corresponding isomer (C') which triggers the orientational alteration of a lot of LC as soldier molecules (S). Accordingly, these processes can be simply expressed as follows; $\mathrm{C} \rightarrow \mathrm{C}^{\prime} \rightarrow \mathrm{n} \times \mathrm{S} \rightarrow \mathrm{n} \times \mathrm{S}^{\prime}$ where $\mathrm{n}$ and $\mathrm{S}^{\prime}$ 'stand for the number of soldier molecules and an LC molecule in a different alignment. It can be reasonable to define $n$ as the amplification degree.

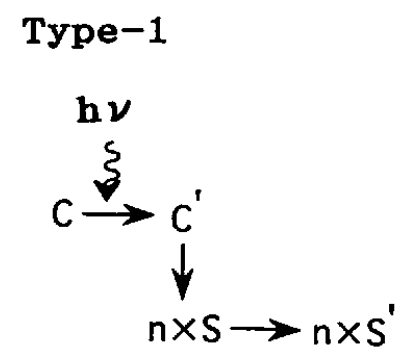

$$
\text { Type-2 }
$$

$$
\text { Type-3 }
$$
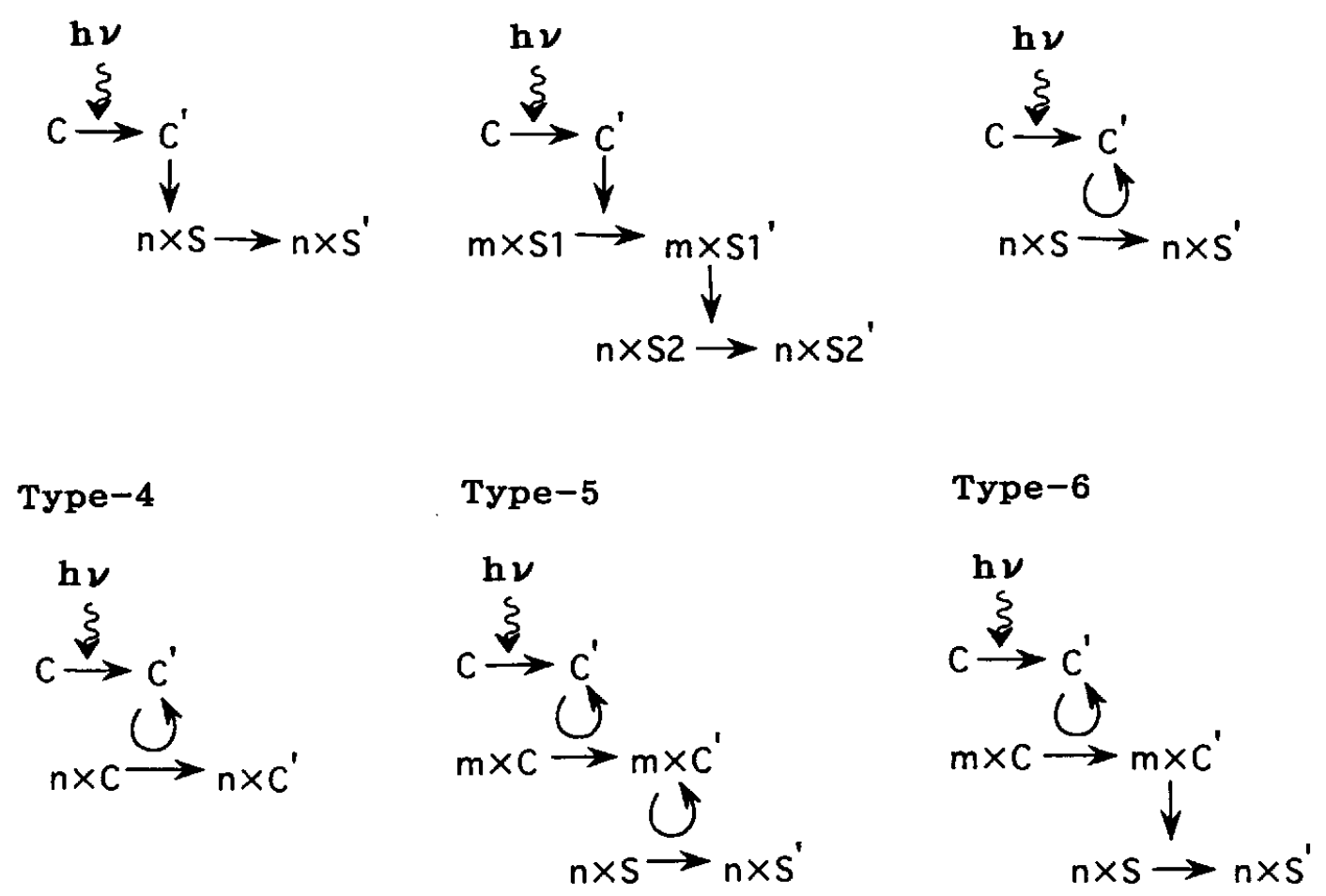

Fig. 1 Molecular amplification schemes.

$\circlearrowleft$ : Catalyzed amplification, $\downarrow$ : Triggered amplification. 
The surface-assisted out-of-plane photoalignment regulation can be also expressed according to the same scheme. Here, $S$ and $S$ ' denote an LC soldier molecule in homeotropic and planar alignment, respectively. The photocontrol of the helical sense of the photosensitive poly(isocyanate) can be also classified into the same type. On the other hand, the type-2 seems to involve the in-plane LC photoalignment. The azimuthal reorientation of LC molecules by the command surface effect may be divided into two processes. The anisotropic orientation of surface azobenzene units induced by linearly polarized light (C $\rightarrow$ C') causes the reorientation of nearest-neighboring LC molecules to a uniaxial alignment at an interface $\left(m \times S 1 \rightarrow m \times S 1^{\prime}\right)$. Subsequently, this two-dimensional rearrangement of LC is transferred to the whole mesophase to give rise to a homogeneous alignment ( $\mathrm{n} \times \mathrm{S} 2 \rightarrow \mathrm{n} \times \mathrm{S2}$ ').

It should be noticed here that there are two kinds of the molecular amplification; triggered amplification and catalytic amplification. The first involves the "domino effect" which is initiated by $C$ ' like a photoisomerized azobenzene and results in modifying the arrangement of molecular aggregation of $S$. The latter is based on a catalytic process to convert $S$ into $S$ '. The crucial difference between them is whether each $S$ has to encounter with C'. The type-3 comprises a chemical reaction ( $\times S \rightarrow n \times S^{\prime}$ ) catalyzed by $C^{\prime}$ which is produced by a photochemical process. A representative system belonging to the type-3 is the chemical amplification photoimaging where $C$ and $C$ ' stand for a photoacid generator and a strong acid, respectively. A molecule acting as a dissolution inhibitor $(S)$ is converted into $S$ ' to cause the solubility change of a polymeric system as a result of the acid catalyzed reaction.

The type-4 is essentially different from the type-3 since the subsequent process takes place autocatalytic. The principle of the silver halide photography may be described by this scheme since photoformed silver atoms (C') in silver grains act as a catalyst for the reduction of silver halide (C) in the development process ( $\mathrm{n} \times \mathrm{S} \rightarrow \mathrm{n} \times \mathrm{S}^{\prime}$ ).

Quite recently, we have reported a novel concept of acid proliferation reactions of simple organic compounds [23]. One of the examples is the decomposition of an acetoacetate derivative having $p$-toluenesulfonyloxy residue catalyzed by p-toluenesulfonic acid (C1') which is produced by the photolysis of a benzoin tosylate ( $1 \rightarrow \mathrm{C} 1$ '). This acid-labile compound (C2) is subjected to the fragmentation reaction catalyzed by the sulfonic acid to produce one more acid molecule ( $2 \rightarrow \mathrm{C} 1$ ') so that the formation of the acid proceeds in a manner of geometric progression. The type-5 has been realized by the 
connection of the acid proliferation reaction to the chemical-amplificationtype photoimaging [24]. In this system, the photolysis of a photoacid generator (C1) yields an acid ( $1^{\prime}$ ) which catalyzes the fragmentation reaction ( $m x$ $\left.\mathrm{C} 2 \rightarrow \mathrm{m} \times \mathrm{C} 1^{\prime}\right)$. The acid acts also as a catalyst for the conversion of a dissolution inhibitor ( $\left.n \times S \rightarrow n \times S^{\prime}\right)$. If the acid proliferation reaction is coupled with cationic polymerization, we may obtain the type-6.

\section{Concluding remarks}

As discussed above, the commander-soldier concept is reasonably adapted to various photopolymeric systems, in particular to photoresponsive materials incorporating photochromic molecules. Surely, this concept might be extended to wide ranges of other photosensitive systems including the visual sensory system initiated by rhodopsin. The photoinduced structural change (C $\rightarrow$ C') of retinylidene residue as a commander unit embedded in the biopolymer causes the structural deformation of the ordered peptide chain as a soldier macromolecule $\left(S \rightarrow S^{\prime}\right)$, leading to subsequent processes in the visual systems. It should be emphasized here that the deformed opsin ( $\left.S^{\prime}\right)$ becomes a commander to cause the subsequent molecular events. In this context, one of the essential points in the "command molecular systems" consists of the conversion of the role of a soldier into a commander which triggers a next conjugated process.

\section{Acknowledgments}

This work was supported by a Grant-in-Aid on Priority-Area-Research "Photoreaction Dynamics" from the Ministry of Education, Science and Culture.

\section{References}

1. K. Ichimura, Kobunshi, 44(1995) 12 .

2. a) K. Ichimura, Y. Suzuki, T. Seki, A. Hosoki and K. Aoki, Langmuir, 4 (1988)1214; b) K. Ichimura, T.Seki, Y. Kawanishi, Y. Suzuki, M. Sakuragi,

T. Tamaki, "Photo-reactive Materials for U1trahigh Density Optical Memory", ed. by M. Irie, Elsevier, Amsterdam (1994)p55.

3. K. Ichimura, "Photochromism: Molecules and Systems", ed. by H. Durr and H. Bouas-Laurent, E1sevier, Amsterdam (1991)p903.

4. E. Sackmann, J. Am. Chem. Soc., 93(1971)7088.

5. T. Ikeda, T. Sasaki and K. Ichimura, Nature, 361(1993) 428.

6. a) T. Ikeda, S. Horiuchi, D. B. Karanjit and S. Tazuke, Macromolecules, 23 (1990)36; b) T. Ikeda, T. Miyamoto, S. Kurihara and S. Tazuke, Mo1. Cryst. 
Liq. Cryst., 188(1990)223; c) T. Ikeda, T. Miyamoto, T. Sasaki, S. Kurihara and S. Tazuke, Mol. Cryst. Liq. Cryst., 188(1990)235.

7. J. H. Wendorff and M. Eich, Mo1. Cryst. Liq. Cryst., 169(1989)133.

8. a) J. Stumpe, L. Muller, D. Kreysig, G Hauck, H. D. Koswig, R. Ruhmann and J. Rubner, Macromo1. Chem., Rapid Commun., 12(1991)81; b) Th. Fischer, L. Lasker, J. Stumpe and S. G. Kostromin, J. Photochem. Photobio1., $80(1994) 453$.

9. K. Anderle, R. Birenheide, M. J. A. Werner and J. H. Wendorff, Liq. Cryst., 9 (1991)691.

10. M. Eich and J. H. Wendorff, Macromo1. Chem., Rapid Commun., 8(1987)467.

11. S. Ivanov, I. Ya. Kovlev, S. Kostromin, V. Shibaev, L. Lasker, J. Stumpe and D. Kreysig, Macromo1. Chem., Rapid Commun., 12(1991)709.

12. K. Ichimura, Y. Hayashi, H. Akiyama and N. Ishizuki, Langmuir, 9(1993) 3298 .

13. W. M. Gibbons, P. J. Shannon, S.-T. Sun and B. J. Swetlin, Nature, 351 (1991) 49 .

14. K. Ichimura, H. Akiyama, N. Ishizuki and Y. Kawanishi, Macromol. Chem., Rapid Commun., 14(1993)813.

15. H. Akiyama, K. Kudo and K. Ichimura, Macromo1. Chem., Rapid Commun., 16 (1995) 35 .

16. Y. Iimura, J. Kusano, S. Kobayashi, T. Aoyagi and T. Sugano, Jpn. J. App1. Phys., Part 2, 32(1993)L93.

17. H. Akiyama, K. Kudo, K. Ichimura, S. Yokoyama, M. Kakimoto and Y. Imai, Langmuir, in press.

18. M. Schadt, K. Schmitt, V. Kozinkov and V. Chigrinov, Jpn. J. App1. Phys., $31(1992) 2155$.

19. T. Ya. Marusii, Yu. A. Reznikov, Mo1. Mater, , 3(1993)161.

20. H. Tomita, K. Kudo and K. Ichimura, unpublished results.

21. M. M. Green, M. P. Reidy, R. J. Johnson, G. Darling, D. J. O'Leary and G. Wilson, J. Am. Chem. Soc., 111(1989)6452.

22. M. Muller and R. Zentel, Macromolecules, 27(1994) 4404.

23. S. Tazuke, S. Kurihara, H. Yamaguchi and T. Ikeda, J. Phys. Chem., 91 (1987) 249 .

24. a) K. Ichimura, K. Arimitsu and K. Kudo, Chem. Lett., submitted; b) K. Kudo, K. Arimitsu and K. Ichimura, Chem. Lett., submitted.

25. K. Arimitsu, K. Kudo, H. Oomori and K. Ichimura, J. Photopolym. Sci. Techno1., submitted. 